\title{
Frequency- and electric-field-dependent conductivity of single-walled carbon nanotube networks of varying density
}

\author{
Hua Xu ${ }^{(a)}$, Shixiong Zhang, and Steven M. Anlage \\ Center for Nanophysics and Advanced Materials, Department of Physics, \\ University of Maryland, College Park, MD 20742-4111 \\ Liangbing $\mathrm{Hu}$ and George Grüner \\ Department of Physics, University of California, Los Angeles, CA 90095
}

\begin{abstract}
We present measurements of the frequency and electric field dependent conductivity of single walled carbon nanotube(SWCNT) networks of various densities. The ac conductivity as a function of frequency is consistent with the extended pair approximation model and increases with frequency above an onset frequency $\omega_{0}$ which varies over seven decades with a range of film thickness from sub-monolayer to $200 \mathrm{~nm}$. The nonlinear electric field-dependent DC conductivity shows strong dependence on film thickness as well. Measurement of the electric field dependence of the resistance $\mathrm{R}(\mathrm{E})$ allows for the determination of a length scale $L_{E}$ possibly characterizing the distance between tube contacts, which is found to systematically decrease with increasing film thickness. The onset frequency $\omega_{0}$ of ac conductivity and the length scale $L_{E}$ of SWCNT networks are found to be correlated, and a physically reasonable empirical formula relating them has been proposed. Such studies will help the understanding of transport properties and benefit the applications of this material system.
\end{abstract}

PACS numbers: $73.63 . \mathrm{Fg}, 72.80 . \mathrm{Ng}$

\section{INTRODUCTION}

Networks with randomly distributed single walled carbon nanotubes (SWCNTs) are emerging as novel materials for various applications, particularly as electronic materials $\frac{1,2,3,4}{1}$ This creates a need for the accurate determination of their fundamental electrical properties.

SWCNT networks can be viewed as a two-dimensional network of conducting one-dimensional rods.$\underline{\underline{5}}$ These rods are either metallic or semiconducting with large dimension ratio(length/diameter $\sim 1000$ ), which leads to the following interesting behavior $\frac{6.7}{.}$ Since the nanotubenanotube junction resistance is much larger than the nanotube resistance itself,,$\frac{7,8,9}{9}$ such a network can be seen as a system with randomly distributed barriers for electrical transport. For a sub-monolayer network, the junction resistance will dominate the overall resistance and the network resistance shows percolation behavior with nonlinear thickness dependence $\underline{\underline{5}, 9}$ For SWCNT networks with thickness in the tens of nanometers, e.g. with tens of layers of SWCNTs, the conductance through the metallic tubes will dominate the conduction, and leads to metallic behavior of the overall resistance $\underline{\underline{10}, 11,12}$

The frequency-dependent and electric-field-dependent conductivity have been investigated for individual SWCNT $13,14.15$ and some types of SWCNT networks $\stackrel{11,16,17,18,19}{ }$ Burke and co-workers measured the microwave conductivity of individual SWCNTs and investigated their operation as a transistor at 2.6 $\mathrm{GHz} \stackrel{13,14}{=}$ For SWCNT networks with $30 \mathrm{~nm}$ thickness,

(a) email: umdxuhua@gmail.com our previous work finds the ac conductivity is frequencyindependent up to an onset frequency $\omega_{0} / 2 \pi$ of about $10 \mathrm{GHz}$, beyond which it increases with an approximate power law behavior $\stackrel{17}{ }$ For thicker films with thickness in the range of tens of micrometers, P. Peit et al. found that conductivities at $\mathrm{DC}$ and $10 \mathrm{GHz}$ are almost the

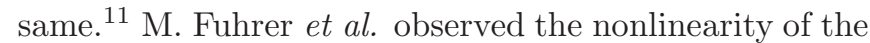
electric field-dependent conductivity of relatively thick nanotube networks, and they claimed that the charge carriers can be localized by disorder in the SWCNTs with an approximate length scale $l_{l o c}$ of $1.65 \mu \mathrm{m}, \frac{18,19}{} \mathrm{~V}$. Skakalova et al. studied the current-voltage $(I-V)$ characteristics of individual nanotubes and nanotube networks of varying thickness, and discussed the modulation of $I-V$ characteristics by a gate-source voltage $\underline{12}$ However there is a lack of frequency and electric field dependent conductivity investigation for systematic variation of network densities, and also there is no correlation study between the onset frequency $\omega_{0}$ and the length scale determined from nonlinear transport.

The frequency- and electric-field-dependent conductivity investigation of different network densities is not only helpful to build a comprehensive understanding of the electrical transport properties of SWCNT networks, but is also important for the sake of applications of these networks $\frac{17,20,21}{2}$ For example, when using SWCNT networks to construct high speed transistors, or as transparent shielding materials, knowledge of their frequency dependent electric transport properties are required. Also use of SWCNT networks as inter-connects in circuits requires understanding of their electric field-dependent properties.

In this paper, we investigated the frequency- and electric-field-dependent conductivity for SWCNT net- 
works with systematically varying densities. We find that the onset frequency $\omega_{0}$ extracted from the frequency dependence measurement increases with the film thickness, while the length scale extracted from the electric field dependent measurement decreases at the same time. Using the measured $\omega_{0}$ and the extracted length scale $L_{E}$ for different films, we developed an empirical relation between the two transport measurement methods.

\section{SAMPLES AND EXPERIMENTAL SETUP}

SWCNT networks on polyethylene terephthalate (PET) substrate are prepared by the spraying method ${ }^{22}$ Arc-discharged nanotube powder from Carbon Solution Inc. was dispersed in water with sodium dodecyl sulphate surfactants. The tubes were purified with nitric acid and left with a variety of functional groups $\stackrel{22}{=}$ After the nanotubes were sprayed on the substrate, the samples were rinsed in water thoroughly to wash away the surfactant. The network density, defined as the number of nanotubes per unit area, was controlled by the solution concentration $(0.1-1 \mathrm{mg} / \mathrm{mL})$ and the spraying time $(10$ - 60 seconds). The nanotubes formed bundles with size of 4-7 nm and length of 2-4 $\mu \mathrm{m}: \underline{23}$ The thicknesses of the films range from submonolayer to $200 \mathrm{~nm}$ corresponding to room temperature sheet conductance ranging from $10^{-6}$ to $10^{-2} \mathrm{~S} /$ Square. Fig. 1] shows SEM images of films with two different coverage densities. It is convenient to characterize the films by their sheet conductance rather than conductivity, because of the inhomogeneous nature of the conducting pathways and thus the difficulty in assigning a film thickness, particularly for submonolayer films.

The Corbino reflectometry setup, which is ideal for broadband conductivity measurements of resistive materials,$\stackrel{24,25,26}{ }$ was used to investigate the conductivity of the SWCNT networks. The measurement procedures are similar to our previous work 17 In order to measure the conductivity in as broad a frequency range as possible, two test instruments are used, the Agilent E8364B network analyzer (covering $10 \mathrm{MHz}$ to $50 \mathrm{GHz}$ ) and the Agilent 4396B network analyzer (covering $100 \mathrm{kHz}$ to 1.8 $\mathrm{GHz}$ ), giving five and half decades of frequency coverage. The electric field dependent conductivity was measured with a standard 4-probe method in a well-shielded high precision DC transport probe $\underline{27}$

\section{FREQUENCY DEPENDENT CONDUCTIVITY}

The data of conductance vs. frequency are shown in Fig. 2(a). The films decrease in thickness from sample 1 (200nm) to sample 5 (sub-monolayer). For all the films, the real parts of the conductance keep their dc value up to a characteristic frequency and start to increase at higher frequency. This kind of behavior has been widely
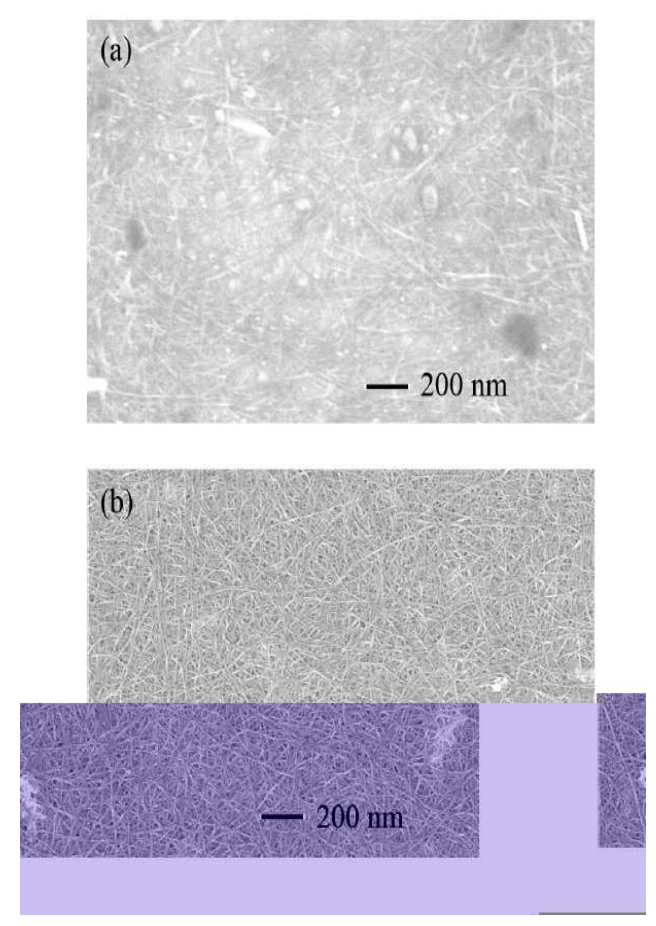

FIG. 1: SEM of two SWCNT network samples with different densities. (a) sub-monolayer nanotube film; (b) nanotube film with thickness about $20 \mathrm{~nm}$.

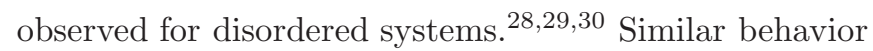
has been found for carbon nanotube polymer composites close to the percolation threshold, in which the extended pair approximation model was applied to describe the observed phenomena $\stackrel{31,32}{=}$ The carbon nanotube networks in Fig. 2(a) have densities well above the percolation threshold. However, since the junction resistances between different tubes are much larger than the resistance of the tubes themselves, $, .8,9,16$ SWCNT networks above the percolation threshold can still be seen as systems with randomly distributed barriers for electrical transport. In this case the extended pair approximation model can be used to describe the real part of the ac conductance:

$$
\sigma=\sigma_{0}\left(1+k\left(\omega / \omega_{0}\right)^{s}\right)
$$

where $\sigma_{0}$ is the dc conductance, $s \leq 1.0, k$ is a constant and $\omega_{0}$ is the onset frequency 31

The obtained frequency dependent conductance in Fig. 2 (a) fit well to the extended pair approximation model. Fig. 2(b) shows the relation between the fit film onset frequency and the dc sheet conductance. The onset frequency changes from $2 \times 10^{6}$ to $1 \times 10^{10} \mathrm{Rad} / \mathrm{Sec}$ as the sheet conductance increases from $10^{-6}$ to $10^{-2} \mathrm{~S} /$ Square. The onset frequency of the ac conductance increases as the dc conductance increases. The solid line Fig. 2. (b) has slope one, implying a linear relation between the onset frequency and their dc sheet conductance, or $\omega_{0} \sim \sigma_{0}$, over about 4 decades. 

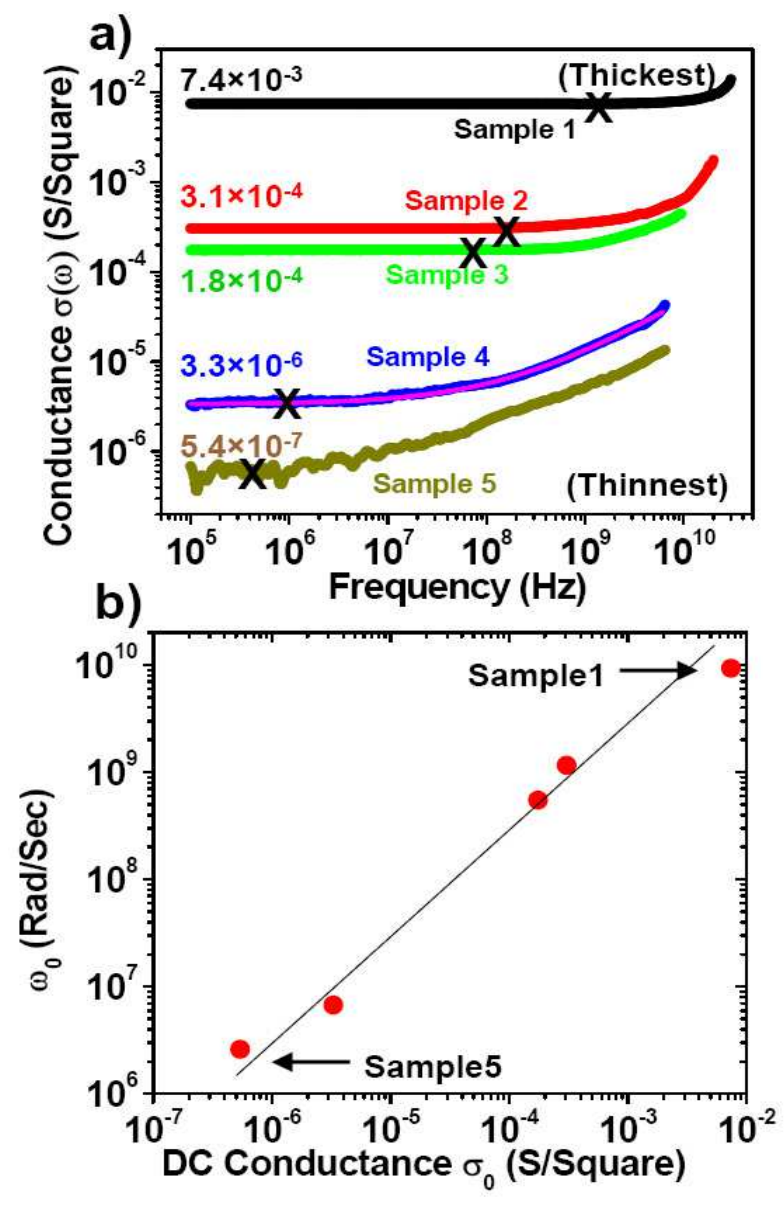

Fig. 3 all have slope one $(\mathrm{Rad} / \mathrm{Sec} \cdot \mathrm{S})$. Here an interesting result is that although the intercepts are very different, the slopes of the three different samples are essentially the same. The difference in intercept is due to the different types of nanotubes. Different carbon nanotube sources have different bundle sizes and lengths, which causes the conductance difference. The polymer-CNT composite has much smaller DC conductance than the other two, due to the separation of SWCNTs by polymer that leads to charge transfer barriers. Despite these differences in detail, there is a universal relationship between onset frequency and dc conductance.

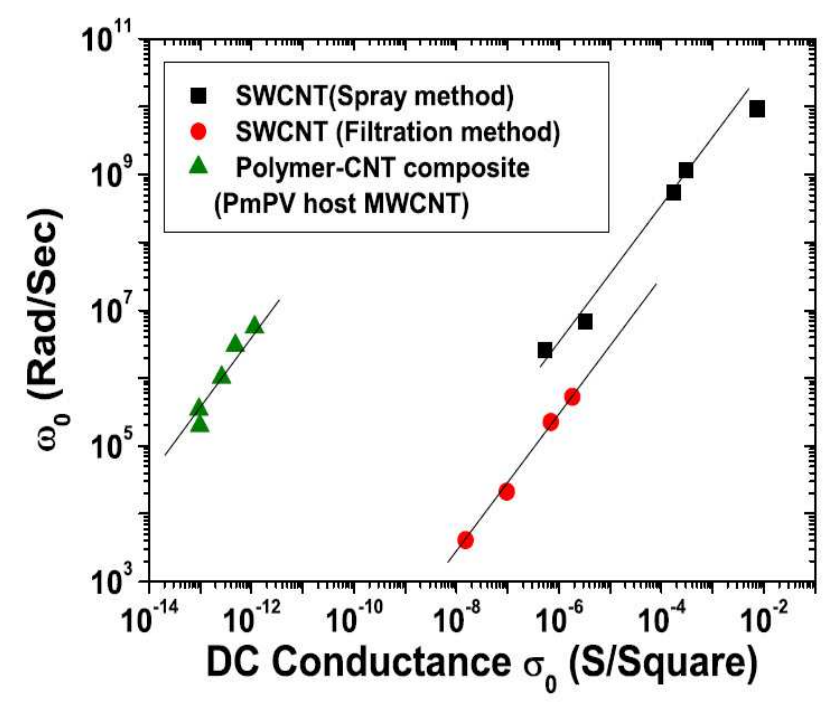

FIG. 2: (Color online) (a) Real parts of the ac conductance for samples with different densities at room temperature. The black crosses mark the fit onset frequency $\omega_{0}$ for each curve. The magenta(grey) line is the typical fit result of the extended pair approximation model for the sample 4 with $\sigma_{0}=3.3 \times 10^{-6} \mathrm{~S} /$ Square, $\omega_{0}=1.1 \times 10^{6} \mathrm{Rad} / \mathrm{Sec}, k=0.12$ and $s=0.52$. (b) The onset frequencies vs. dc conductance of SWCNT networks. The solid line has slope 1.

We also measured the frequency-dependent conductance of ultra-thin sub-monolayer nanotube networks, which are fabricated via the filtration method. $\stackrel{5}{*}$ We choose Chloroform as the solvent instead of water to avoid the washing steps which can easily destroy the submonolayer film structure. The network density is controlled by the concentration and volume of the solvent used $\underline{\underline{5}}$ The conductance of these films are measured by a $4284 \mathrm{~A}$ Precision LCR Meter, covering the frequency range from $20 \mathrm{~Hz}$ to $1 \mathrm{MHz}$. For these films just above the percolation threshold, their frequency dependent conductance also fit well to the extended pair approximation model. In Fig. 3, we plot the onset frequency versus their dc sheet conductance. For comparison, we also present

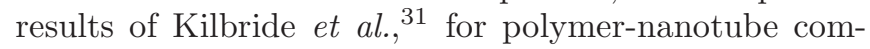
posites as well as the data from Fig. 2(b) for our films prepared by the spray method. The three solid lines in

FIG. 3: (Color online) The onset frequency vs. dc conductance for different SWCNT networks at room temperature. The three solid lines all have slope one.

The linear proportionality $\omega_{0} \sim \sigma_{0}$ is one of the characteristic features of ac conductance of disordered solids $\underline{30}$ For disordered systems, the universality of ac conductance has been studied by many researchers since its discovery in the 1950's, and is usually referred to as TaylorIsard scaling. $28,29,30$ This inspires us to investigate the scaling behavior of the conductance and frequency for SWCNT networks. Similar to Kilbride et al.'s work ${ }^{31}$, taking the dc conductance $\sigma_{0}$ and the onset frequency $\omega_{0}$ as scaling parameters, we plotted the ac conductance data for all samples in Fig. 4. The data show a scaling behavior with all data sets falling on the same master curve described by $k=0.12$ and $s=0.52$.

In order to explain the observed onset frequency change with the SWCNT volume fraction for polymernanotube composites close to percolation threshold, Kilbride et al. defined a characteristic length scale, the "correlation length" $\xi \stackrel{31}{\underline{31}}$ For our samples, which are well above the percolation threshold, we can similarly define the correlation length $\xi$ as the distance between connections in the sample, i.e., the distance between junctions of the multiply-connected SWCNT network. When one 


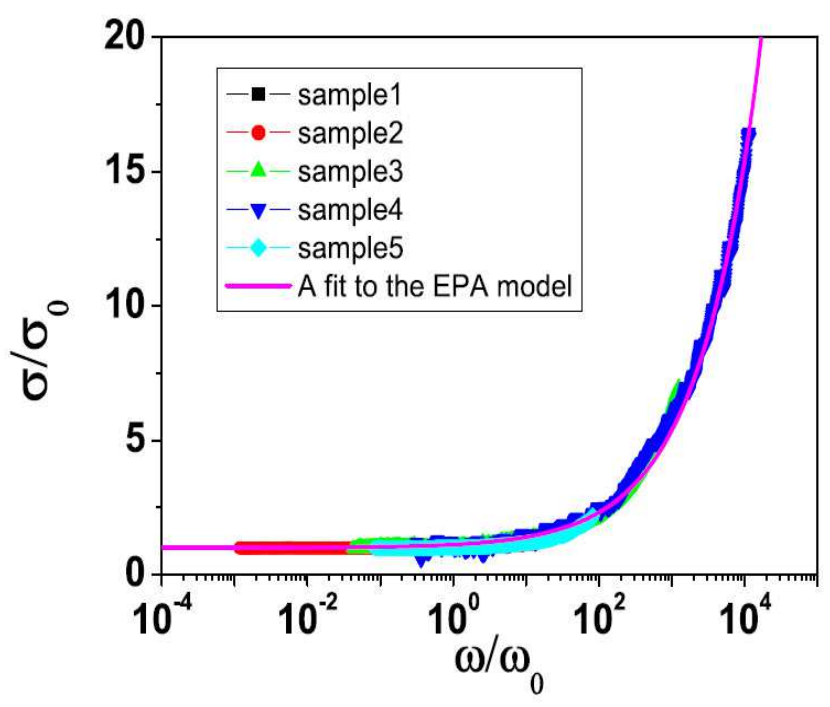

FIG. 4: (Color online) Master scaling curve showing the ac conductance for SWCNT network samples(spray method) with different densities at room temperature. The red(grey) solid line is a fit to the extended pair approximation model $k=0.12$ and $s=0.52$.

measures the conductance at a given frequency, there is a typical probed length scale. At low frequencies (corresponding to long time periods), the carriers travel long distances in one-half ac cycle and the experiment investigates longer length scales. At high frequencies, the carriers travel short distances in one half ac-cycle and the probed length scale is shorter. In the absence of an applied DC electric field, and assuming a random walk, the probed length scale $L_{\omega} \propto \omega^{-1 / 2}, 31$

For low frequency, $L_{\omega}>\xi$, the probed length scale spans multiple junctions of the SWCNT network. The junction resistances between different tubes are much larger than the resistances of the tubes themselves, $\underline{7,8,9,16}$ Hence the conductance is small and equal to the dc conductance. As the frequency increases, $L_{\omega}$ becomes smaller than $\xi, L_{\omega}<\xi$, and the junction resistances have less contribution to the total resistance. The intrinsic properties of the SWCNT then dominate the measurement. Therefore the observed conductance increases as the frequency increases. The transition frequency is expected to be that where carriers scan an average distance of order of the correlation length. $\frac{31}{}$

The SWCNT film can be treated as a random walk network, in which the probed length scale at a given frequency goes as $L_{\omega} \sim \omega^{-1 / 2}$. Then with the onset frequency $\omega_{0}$ of samples we are able to estimate their correlation length $\xi \simeq L_{\omega_{0}} \propto \sqrt{\frac{1}{\omega_{0}}}$. As the density of the SWCNT networks increases, there are more junctions and the average distance between connections, the correlation length $\xi$, becomes smaller and thus the onset frequency $\omega_{0}$ increases. This is consistent with the behavior shown by the data in Fig. 2

\section{ELECTRIC FIELD DEPENDENT CONDUCTIVITY}

From the measured frequency dependent conductivity, we observed that the correlation length $\xi$ varies as the thickness of the film increases. Due to the large resistance of junctions between different tubes, the carriers in the SWCNT networks are easier to move on small length scales than on larger length scales. This phenomenon inspires us to investigate whether the transport properties are nonlinear as a result. According to the works of M. S. Fuhrer et al., the localization behavior of a SWCNT network can be studied by measuring the electric field dependent nonlinearity of the conductivity $\stackrel{18,19}{ }$ Generally, increasing both the temperature and the electric field reduces the effects of localization. Hence a system typically displays a characteristic electric field at which nonlinear conductivity begins to appear. Through measurement of the characteristic field $E_{c}$ one can determine a length scale of the system:

$$
L_{E}=\frac{k_{B} T}{e E_{c}}
$$

where $L_{E}$ corresponds to the size of the regions with good conductivity, and $e E L_{E}$ is the energy gained by a carrier in one conducting region on average 33,34 . For our SWCNT films $L_{E}$ depends on the average distance between connections and thus should vary with sample thickness. For relatively thick films $(\sim 10 \mu \mathrm{m})$ with low sheet resistance, Fuhrer et al. found a temperatureindependent length scale $l_{l o c} \sim 1.65 \mu \mathrm{m}$ in a SWCNT film. 18,19

Here we investigated the electric field-dependent conductance for a different class of films. The studied samples were cut into rectangular pieces with length $\sim 8 \mathrm{~mm}$ and width $\sim 3 \mathrm{~mm}$. Gold contact leads, which completely cover the width of the samples, were deposited on the surfaces of the samples to form a standard four probe pattern. The measured SWCNT networks have dimensions of $\sim 3.5 \mathrm{~mm} \times 3 \mathrm{~mm}$, between the two voltage leads. Because SWCNT films have a large in-plane thermal conductivity $, 35,36$ and the probe used to perform the measurement was specially designed to reduce heating effects 27 we believe that the heating effect can be ignored in our measurement range. This was confirmed by observing the voltage response of pulsed current input of several samples in the time domain with an oscilloscope.

The DC resistance vs. electric field of a submonolayer film(sample 5) at different temperatures is shown in Fig. 5 (a). We can see clearly that there is nonlinear behavior even at room temperature. As sketched in Fig. 5(a), we extracted $E_{c}$ for each temperature, which is the characteristic electric field at which nonlinear conductance begins to appear $\stackrel{18}{18}$ Fig. 5 (b) shows the extracted $E_{c}$ vs temperature for this sample. As temperature increases, $E_{c}$ also increases. Roughly, $E_{c}$ and temperature have a linear relationship and satisfy the equation $E_{c}=k_{B} T / e L_{E}$, with $L_{E} \approx 54 \mu \mathrm{m}$. Note that this is much larger than the 


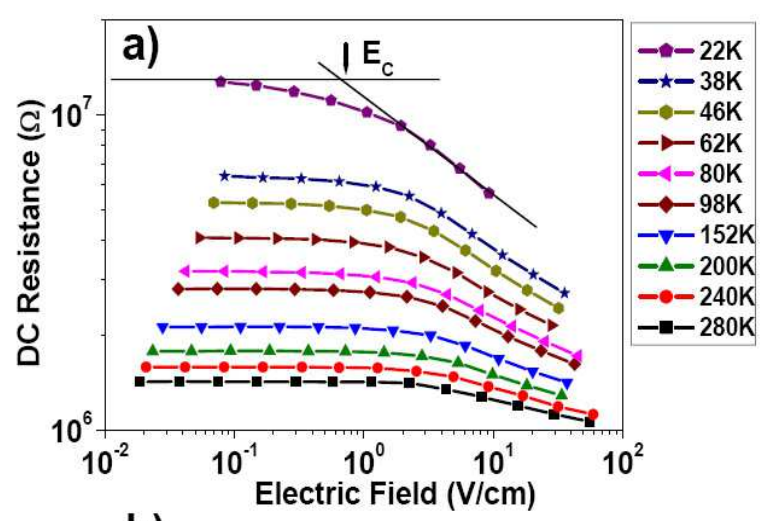

b)

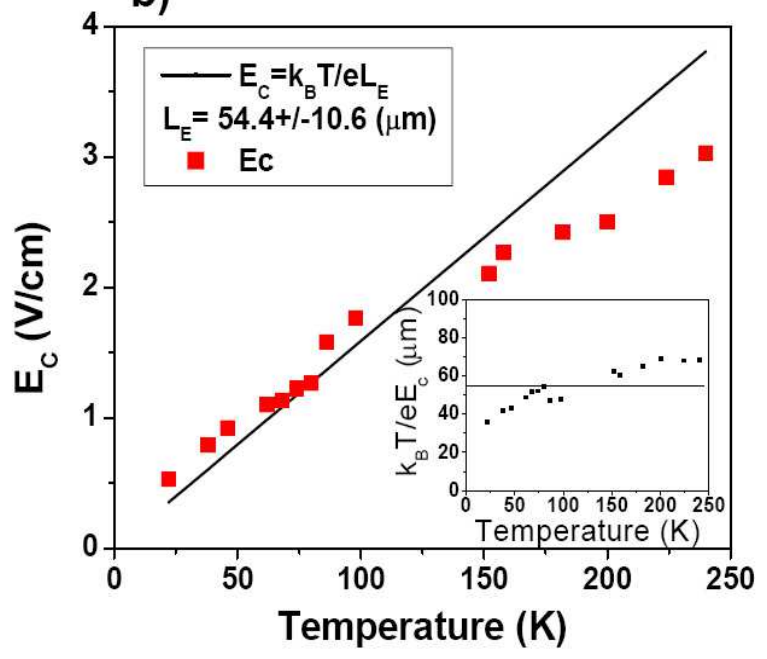

FIG. 5: (color online) (a) DC resistance vs electric field for a submonolayer(sample 5) SWCNT network at different temperatures. (b) $E_{c}$ vs temperature and extracted length scale for this sample.

$l_{\text {loc }}$ obtained in Ref ${ }^{18,19}$. This suggests that a different mechanism for hindering transport is active here, namely poor contacts between bundles of SWCNTs.

To investigate the dependence of $L_{E}$ on the film thickness, we measured the electric field-dependent conductance for samples with various thicknesses. We found that the nonlinear behavior strongly depends on the thickness of the film. At low temperature, all the samples show nonlinear behavior of resistance on electric field, and the critical field $E_{c}$ is larger for thicker samples and smaller for thin samples. At room temperature, all the other samples are purely ohmic(linear) in electric fields up to $100 \mathrm{~V} / \mathrm{cm}$, except for the thinnest sample, sample 5, which showed electric field-dependent conductance at all temperatures. Through the critical field $E_{c}$, we extracted the $L_{E}$ for different density films, shown in Fig. [ 6 as red-dots. Clearly $L_{E}$ depends on the density of the SWCNT networks. For the thicker film with larger SWCNT density, which has larger sheet conductance, the $L_{E}$ is smaller. The lower density films with larger average distance between the junctions has larger $L_{E}$.

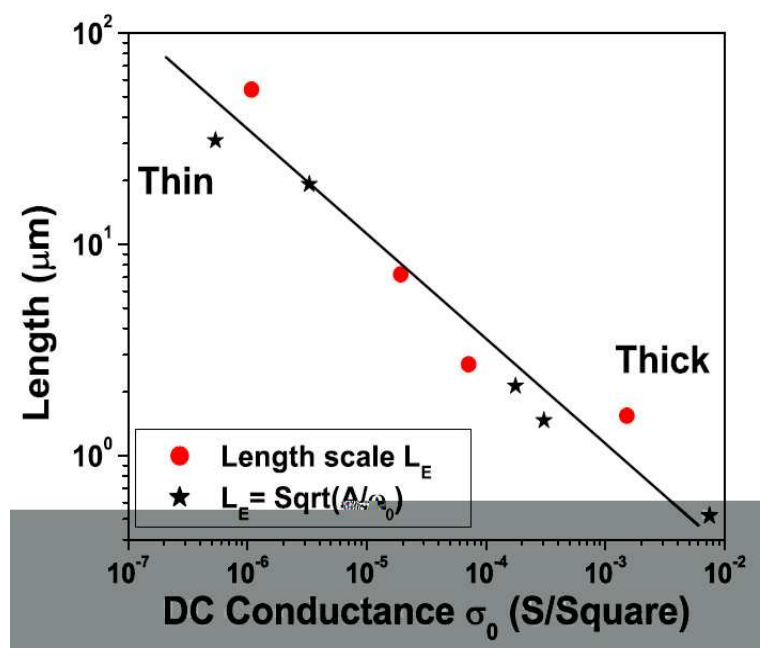

FIG. 6: (Color online) Comparison of the length scale $L_{E}$ obtained from electric field dependent DC conductance data(red dot), and the estimated length scale from frequency dependent conductance measurement $\sqrt{A / \omega_{0}}$ (black star) of SWCNT networks of various densities. The line has slope $-1 / 2$ in the log-log plot.

Although the localization behavior may be affected by the properties of individual SWCNTs 18,19 , the above result shows that the observed behavior is more likely a consequence of poor electric conduction through interbundle junctions. As the thickness and density increase, there are more and more connections, which decreases the length scale $L_{E}$, as shown in Fig. 6]

When the film thickness increases to a large value, the density of the SWCNTs and connections of the film will finally saturate and stop increasing. For those very thick SWCNT films (also called mats), their correlation length $\xi$, and also the length scale $L_{E}$, as well as the onset frequency, will be independent of the thickness, but depend on the properties of individual bundles, which include both the disorder of the individual nanotube and also the geometry of the bundles. Hence the difference of SWCNT sources and purities can change the onset frequency. We believe this is the reason that our previous work found that the ac conductivity follows the extended pair approximation model with an onset frequency $\omega_{0}$ about $10 \mathrm{GHz}$, while Peit et al. found that SWCNT networks have the same conductivities at DC and $10 \mathrm{GHz}$ for SWCNT networks with thickness in the range of tens of micrometers $\stackrel{11,17}{\underline{1}}$

\section{DISCUSSION}

The frequency dependent conductivity shows that the onset frequency $\omega_{0}$ increases with the sample thickness and gives an estimation of the correlation length $\xi$ of 
the sample, $\xi \sim L_{\omega_{0}} \propto \sqrt{\frac{1}{\omega_{0}}}$. $\xi$ and $L_{\omega_{0}}$ decrease as the sample thickness increases. The DC nonlinear conductivity measurement shows that the length scale $L_{E}$ of the samples also decreases with increasing sample thickness. Qualitatively $L_{\omega_{0}}$ and $L_{E}$ have the same dependence on the sample thickness. Based on the above consideration, it is reasonable to expect some correlation between the onset frequency $\omega_{0}$ and the length scale $L_{E}$, and we proposed the following relation,

$$
L_{E}=\sqrt{\frac{A}{\omega_{0}}},
$$

where $A$ is a fitting parameter, which can be obtain from our experiment data, $A \sim 2.5 \pm 0.5 \times 10^{-3} \mathrm{~m}^{2} / \mathrm{s}$. Fig. [6 shows the comparison of the nonlinear transport length scale $L_{E}$ measured directly through electric-field dependent DC conductance measurements(red-dot) and the right-hand side of Eq. 3 from the frequency dependent measurement(black-star). They are consistent with each other. In this way we obtain an empirical relation between the frequency-dependent and the electric fielddependent conductivity. The parameter $A$ should depend on the diffusion properties of carriers in the SWCNT films $\frac{37}{3}$ Writing $L_{E}=\sqrt{4 D t_{*}}$, where $\mathrm{D}$ is a diffusion constant and $t_{*}$ is the corresponding time scale, we take $D=A / 8 \pi$. Assuming the Einstein relation $\mu=\frac{D e}{k_{R} T}$, we can estimate the mobility of carriers on the SWCNT network, which is on the order of $10^{-2} \mathrm{~m}^{2} / \mathrm{V}$. sec. This is much smaller than that of the individual single walled carbon nanotube, which can be on the order of $10^{0}-10^{1} \mathrm{~m}^{2} / \mathrm{V} \cdot \mathrm{sec}$ at room temperature $\frac{38,39}{\mathrm{In}}$ the SWCNT networks, due to the randomly distributed barriers for electrical transport, the mobility is expected to be much smaller than the SWCNT itself. The carbon nanotube film-based transistors have reported mobilities on the order of $10^{-4}-10^{-1} \mathrm{~m}^{2} / \mathrm{V} \cdot \sec , 20.40,41$ which is consistent with our estimation. To get a deeper physical understanding of these networks, more investigation and theoretical work are needed. For example, measuring the temperature dependence of the frequency-dependent conductivity for SWCNT networks with various densities would be one approach: $\underline{11,17}$

\section{CONCLUSION}

To conclude, we systematically studied the frequency and electric field dependent conductivity of SWCNT films of various thicknesses. We found the poor interbundle junctions affect the transport behavior of the carriers in the SWNCT films, which causes strong thickness dependence of characteristic length scales. A thinner film has larger correlation length $\xi$ and length scale $L_{E}$, so it has a smaller onset frequency $\omega_{0}$, whereas a thicker film with smaller correlation length $\xi$ and length scale $L_{E}$, has a larger onset frequency $\omega_{0}$. An approximate empirical formula relating the onset frequency $\omega_{0}$ and nonlinear transport length scale establishes contact between the frequency and the electric field dependent conductivity, which helps us to understand the electric transport properties of the SWCNT films.

The authors would like to thank M. S. Fuhrer, C. J. Lobb, Weiqiang Yu and Bing Liang for their help and discussions on this work. This work has been supported by Center for Nanophysics and Advanced Materials of the University of Maryland and the National Science Foundation Grant Nos. (DMR-0404029), (DMR-0322844), and (DMR-0302596).
${ }^{1}$ George Grüner, J. Mater. Chem. 16,3533 (2006).

2 P. M. Ajayan and O. Z. Zhou, Carbon Nanotubes: Synthesis, Structure, Properties and Application, Springer: Berlin/Heidelberg, 2001.

3 Ray H. Baughman, Anvar A. Zakhidov and Walt A. de Heer, Science 297, 787 (2002).

4 D. Zhang,K. Ryu, X. Liu, E. Polikarpov, J. Ly, M. E. Tompson and C. Zhou, Nano Letters, 6, 1880, (2006).

${ }^{5}$ L. Hu, D.S. Hecht and G. Grüner, Nano Lett., 4, 2513, (2004).

6 M. S. Dresselhaus, G. Dresselhaus, P. C. Eklund, Science of Fullerenes and Carbon Nanotubes Academic Press, San Diego, CA (1996).

7 M. S. Fuhrer, J. Nygard, L. Shih, M. Forero, T. Yoon, M. S. C. Mazzoni, H. J. Choi, J. Ihm, S. G. Louie, A. Zettl and P. L. Mceuen, Science 288, 494, (2000).

8 A. B. Kaiser, G. Dusberg and S. Roth, Phys. Rev. B 57, 1418, (1998).

9 M. Stadermann, S. J. Papadakis, M. R. Falvo, J. Novak, E. Snow, Q. Fu, J. Liu, Y. Fridman, J. J. Boland, R. Superfine, and S. Washburn, Phys. Rev. B 69, 201402(R)
(2004).

10 J. E. Fischer, H. Dai, A. Thess, R. Lee, N. M. Hanjani, D. L. Dehaas and R. E. Smalley, Phys. Rev. B 55, R4921, (1997).

11 P. Petit, E. Jouguelet, J. E. Fischer, A. G. Rinzler and R. E. Smalley, Phys. Rev. B 56, 9275, (1997).

12 V. Skakalova, A. B. Kaiser, Y. S. Woo and S. Roth, Phys. Rev. B 74, 085403, (2006).

13 Z. Yu and P. J. Burke, Nano Lett. 5, 1403 (2005); S. Li, Z. Yu, S. Yen, W. C. Tang and P. J. Burke, Nano Lett. 4, 753 (2004).

14 P. J. Burke, IEEE Trans. on Nano. Tech. 2, 55 (2003).

15 M. Zhang, X. Huo, P. Chan, Q. Liang and Z. K. Zhang, Appl. Phys. Lett. 88163109 (2006).

16 A. B. Kaiser, K. J. Challis, G. C. Mcintosh, G. T. Kim, H. Y. Yu, J. G. Park, S. H. Jhang and Y. W. Park, Curr. Appl. Phys. 2, 163 (2002).

17 Hua Xu, L. Hu, S. M. Anlage and G. Grüner, Appl. Phys. Lett. 90, 183119 (2007).

18 M. S. Fuhrer, M. L. Cohen, A. Zettl, V. Crespi, Solid. State Comm. 109, 105 (1999). 
19 M. S. Fuhrer, W. Holmes, P. L. Richards, P. Delaney, S. G. Louie and A. Zettl, Synthetic Metals 103, 2529 (1999).

20 S.J. Kang, C. Kocabas, T. Ozel, M. Shim, N. Pimparkar, M.A. Alam, S.V. Rotkin and J.A. Rogers, Nature Nanotechnology 2, 230 (2007).

21 Z. Wu, Z. Chen, X. Du, J. M. Logan, J. Sippel, M. Nikolou, K. Kamaras, J. R. Reynolds, D. B. Tanner, A. F. Hebard and A. G. Rinzler, Science 305, 1273 (2004).

22 E. Bekyarova, M. E. Itkis, N. Cabrera, B. Zhao, A. Yu, J. Gao and R. C. Haddon, J. Am. Chem. Soc. 127, 5990 (2005).

23 L. Hu, G. Grüner, J. Gong, C-J Kim, B. Hornbostel, Appl. Phys. Lett., 90, 093124 (2007).

24 J. C. Booth, Dong Ho Wu, and Steven M. Anlage Rev. Sci. Instrum., 65, 2082 (1994).

25 J. C. Booth, D. H. Wu, S. B. Qadri, E. F. Skelton, M. S. Osofsky, A. Pique, and S. M. Anlage, Phys. Rev. Lett. 77, 4438 (1996).

26 A. Schwartz, M. Scheffler, S. M. Anlage, Phys. Rev. B 61, R870, (2000).

$27 \mathrm{Su} \mathrm{Li}$, Phase Transitions of High-Temperature Superconductors, Ph. D. thesis, University of Maryland, 2007.

28 Jeppe C. Dyre J. Appl. Phys. 64, 2456 (1988).

29 P. Dutta, S. Biswas, M. Ghosh, S. K. De and S. Chatterjee, Synthetic metals 122, 455 (2001).
30 Jeppe C. Dyre and Thomas B. Schroder, Rev. Mod. Phys. 72, 873 (2000).

31 B. E. Kilbride, J. N. Coleman, J. Fraysse, P. Fournet,M. Cadek, A. Drury, S. Hutzler, S. Roth and W. J. Blau, J. Appl. Phys. 92, 4024, (2002).

32 D. S. Mclachlan, C. Chiteme, C. Park, K. E. Wise, S. E. Lowther, P. T. Lillehei, E. J. Siochi and J. S. Harrison, J. of Polymer science, B 43, 3273 (2005).

33 K. Mortensen, M. L. W. Thewalt, Y. Tomkiewicz, T. C. Clarke, G. B. Street, Phys. Rev. Lett. 45, 490 (1980).

34 N. Apsley and H. P. Hughes, Phil. Mag. 31, 1327 (1975).

35 J. Hone, M. Whitney, C. Piskoti, and A. Zettl, Phys. Rev. B 59, R2514 (1999).

36 Y. Ouyang and J. Guo, Appl. Phys. Lett. 89, 183122 (2006).

37 P. Minnhagen Rev. Mod. Phys. 59, 1001 (1987).

38 M. S. Fuhrer, B. M. Kim, T. Dürkop, and T. Brintlinger, Nano Lett. 2, 755 (2002).

39 T. Dürkop, B. M. Kim, and M. S Fuhrer, J. of Phys.: Cond. Matt. 16, R553 (2004).

40 E. Artukovic, M. Kaempgen, D. S. Hecht, S. Roth and G.Grüner, Nano Letters, 5, 757, (2005).

41 W. Xue and T. Cui, Appl. Phys. Lett. 89, 163512 (2006). 